ORIGINAL ARTICLE

\title{
Preconsultation use of analgesics on adults presenting to the emergency department with acute appendicitis
}

\author{
C-F Chong, T-L Wang, C-C Chen, H-P Ma, H Chang
}

Emerg Med J 2004;21:41-43

See end of article for authors' affiliations

......................

Correspondence to: Dr C-F Chong, Emergency Department, Shin Kong Wu Ho-Su Memorial Hospital, 95 Wen Chang Road, Taipei, 111 Taiwan; jackchong@tmu.edu.tw

Accepted for publication 27 March 2003

\begin{abstract}
Objective: 279 cases of appendicitis were reviewed and compared for the difference between those patients who received pain medication before consulting a surgeon and those who were not treated with analgesics.

Methods: All patients aged 15 years and older who underwent appendicectomy for appendicitis between 1 July 2001 and 30 June 2002 were divided into group 1 (those who received preconsultaion use of analgesics) and group 2 (those who were not treated with analgesics). The following measures were compared: age, sex, symptom duration, initial vital signs, white blood cell counts, frequency of imaging studies, time to operative intervention, and operative findings. Continuous and categorical variables were analysed using $t$ and $\chi^{2}$ tests, respectively.

Results: A total of 279 patients were included for analysis. Patient details (age, sex, symptom duration) of the two study groups were similar. There was no statistically significant difference between group 1 and group 2 with respect to vital signs (systolic blood pressure, pulse rate, respiratory rate, body temperature), white blood cell counts, and frequency of imaging studies (ultrasound, computed tomography). There was no significant difference in the rate of perforated appendicitis between the two study groups although a shorter median time to operative intervention has been found in the group who received analegesia. Conclusion: The preconsultation use of analgesics in ED patients with a final diagnosis of appendicitis is not associated with a longer delay to operative intervention and is not associated with an increased rate of perforated appendicitis.
\end{abstract}

A ppendicitis is a common cause of acute abdominal pain. ${ }^{1}$ Most patients present initially to the emergency department (ED). Because of its highly variable historical and physical manifestations and its inconsistent course, delayed or missed diagnosis of appendicitis continues to be one of the most frequent causes of medical malpractice claims and settlements for emergency physicians. ${ }^{23}$ Early administration of analgesics in this group of patients might have confused the picture further.

In the past, early analgesia was considered inadvisable as it could mask physical signs and hence delay diagnosis and intervention. ${ }^{4}$ Recently, this point of view has been challenged. Pain control is at present considered an integral part of patient resuscitation as it minimises stress responses and facilitates patient cooperation during physical examination..$^{5-7}$ Although more and more authors argue that early pain relief is safe in abdominal pain patients, many practising surgeons still believe that pain drugs would interfere with diagnostic accuracy. ${ }^{8}$ Various studies attempted to assess the safety of analgesia in abdominal pain patients with clinical end points mostly focused on the effects of analgesia on abdominal signs, diagnosis confidence, management, and disposition decisions from the points of view of the operating surgeons. In this study, we evaluated the effects of analgesia prescribed by emergency physicians before consultation with surgeons on the outcomes (time to surgery and the rate of perforated appendicitis) of patients presented with appendicitis.

The basic pathophysiology of appendicitis is obstruction of the lumen of the appendix followed by intraluminal hypertension, ischaemia of the appendiceal wall, and bacterial invasion. Without interruption, this process will usually lead to gangrene and perforation. Although not proved, it is believed that delay in diagnosis and treatment is associated with increased risk of perforation. ${ }^{9}$ Our study hypothesis is that patients with appendicitis who received early pain control experience a longer delay to operative intervention resulting in an increased rate of perforated appendicitis. Our study was designed to examine whether there is significant difference in the time to operative intervention and in the rate of perforated appendicitis between patients who received preconsultation use of analgesics and those who were not treated with analgesics.

\section{METHODS}

The study was conducted in Taiwan at Shin Kong Wu Ho-Su Memorial hospital, which provides urban tertiary care with an annual ED census of 84000 . This study used a retrospective chart review design. All patients aged 15 years and older who underwent appendicectomy for appendicitis between 1 July 2001 and 30 June 2002 were divided into groups 1 (those received preconsultation use of analgesics) and 2 (those who were not treated with analgesics). All ED and inpatient records along with operative reports and pathological reports were reviewed by one of two researchers and data collected on standardised forms. All patients who had been treated with parenteral (intravenous or intramuscular) non-steroidal anti-inflammatory drugs (NSAIDs) or narcotics before consulting a surgeon were classified into group 1. Parenteral NSAIDs available to our ED during the study period were ketoprofen (Profenid) $100 \mathrm{mg}$ IV/IM and ketorolac (Keto) $60 \mathrm{mg}$ IV/IM; and parenteral narcotic analgesics were morphine $0.1 \mathrm{mg} / \mathrm{kg}$ IV/IM, and meperidine $1 \mathrm{mg} / \mathrm{kg}$ IV/IM. Antispasmodic agents such as hyosine-N-

Abbreviations: $E D$, emergency department; $\mathrm{WBC}$, white blood cell $\mathrm{SBP}$, systolic blood pressure; $\mathrm{PR}$, pulse rate; $\mathrm{RR}$, respiratory rate; $\mathrm{BT}$ body temperature; CT, computed tomography; NSAIDs, non-steroidal anti-inflammatory drugs 
butylbromide (Buscopan) were not considered as "analgesics" in our study.

Initial data collected for comparison were patient characteristics (age, sex, duration of symptoms since onset), vital signs (systolic blood pressure (SBP), pulse rate (PR), respiratory rate $(R R)$, body temperature $(B T))$, and white blood cell (WBC) counts. The number of patients in each group who required further imaging study with ultrasound or computed tomography (CT) were also recorded. The outcome variables that we wanted to compare between the two groups were the time from ED presentation to operative intervention and the rate of perforated appendicitis. Data of initial SBP, $\mathrm{PR}, \mathrm{RR}$, and BT were collected by the ED triage nurse. Data of initial WBC counts and records of imaging studies (ultrasound or CT) were obtained through the hospital information system.

Continuous variables were presented as mean (SD) or median (interquartile (25th to 75 th) range) and compared using the independent samples $t$ test (assuming normal distribution) or the Mann-Whitney and Wilcoxon tests (assuming non-normality). Categorical variables were compared using the $\chi^{2}$ test. All analyses were performed on SPSS 10.0 for Windows. Statistical significance was set at $\mathrm{p}<0.05$ (two tailed).

\section{RESULTS}

A total of 310 patients were identified with a final hospital discharge diagnosis of appendicitis using retrospective chart review. Six cases were excluded because of incomplete chart records. Eight were excluded because of presentation from the outpatient department. Fifteen were excluded because appendicectomy was performed incidental to another procedure. Two were excluded because they were haemodynamically unstable ( $\mathrm{SBP}<90 \mathrm{~mm} \mathrm{Hg}$ ) on presentation to the ED.

During the study period, a total of 279 patients were included in the study population. The mean age was 37 years and the sex distribution is nearly equal ( $52.7 \%$ male). Most patients $(78.1 \%)$ presented to the ED with symptoms less than two days. The most commonly ordered laboratory test was a WBC count soon after arrival at the ED (mean initial WBC count was $13.2 \mathrm{k} / \mathrm{mm}^{3}$ ). An ultrasound study was needed in $18.3 \%$ of the cases, and CT scans were needed in $7.9 \%$ of the cases. Of the 279 patients comprising the study population, 115 patients were treated with parenteral analgesics before consulting a surgeon (group 1) and 164 patients did not receive pain control before surgical consultation (group 2). As shown in table 1, there was no significant difference between the two study groups in mean age, sex distribution, and proportion of patients with symptoms $>48$ hours. Table 2 showed that group 1 and group 2 patients had insignificant difference in their initial vital signs ( $\mathrm{SBP}, \mathrm{PR}, \mathrm{RR}$, and $\mathrm{BT}$ ), initial WBC counts, and frequency of imaging studies (ultrasound or CT). The mean (SD) time to operative intervention was 831.6 (582.5) minutes for group 1 and 1134.2 (605.0) minutes for group $2(p=0.71)$. The median time to surgery was $390(259-608)$ minutes in group 1 and 545 (303-913) minutes in group 2 $(\mathrm{p}=0.001)$. Perforated appendicitis was identified in 25 patients $(21.7 \%)$ from group 1 and in 35 patients $(21.3 \%)$ from group $2(p=0.88)$. These data are summarised in table 3 .

\section{DISCUSSION}

The use of analgesics by emergency physicians before surgical consultation in ED patients who present with abdominal pain has remained controversial. Pain relief is often postponed for fear that it may mask the physical signs and interfere the diagnosis and treatment of these patients. ${ }^{10}$ It was suggested in several previous studies that analgesic use is safe in the diagnosis of acute abdominal pain including acute appendicitis. $^{4-6}$ Some authors even indicated that early pain relief actually facilitates diagnosis of acute abdominal pain. ${ }^{11}{ }^{12}$

In this cohort of patients studied, the two study groups had similar patient characteristics and comparable preoperative vital signs-there was no statistically significant difference between groupl and group 2 with respect to their age, sex, symptom duration, and initial SBP, PR, RR, and BT. The severity of disease on presentation was also comparable between the two groups of patients because indicators of complicated appendicitis (including the WBC counts, frequency of imaging studies, and the proportion of patients with symptom duration $>48$ hours) were similar for both groups.

The study hypothesis is that patients with appendicitis who received preconsultation use of analgesics (group 1) experience a longer delay to operative intervention resulting in an increased rate of perforated appendicitis. However, this hypothesis is rejected by our study. We found no difference in the rate of perforated appendicitis and the mean time to surgery between the two groups though the median time to

\begin{tabular}{|c|c|c|c|c|}
\hline & Total $(n=279)$ & Group $1(n=115)$ & Group $2(n=164)$ & p Value \\
\hline Age (SD), y & 37 (15) (range 17-80) & 38 (14) (range 17-79) & 37 (15) (range 17-80) & 0.35 \\
\hline Male (\%) & $147(52.7)$ & $62(53.9)$ & $85(51.8)$ & 0.85 \\
\hline Symptoms > 48 h (\%) & $61(21.9)$ & $23(20.0)$ & $38(23.2)$ & 0.65 \\
\hline
\end{tabular}

\begin{tabular}{|c|c|c|c|c|}
\hline & Total $(n=279)$ & Group $1(n=115)$ & Group $2(n=164)$ & p Value \\
\hline SBP (SD) mm Hg & $123(19)$ & $122(19)$ & $123(19)$ & 0.83 \\
\hline $\mathrm{PR}(\mathrm{SD}) / \mathrm{min}$ & $82(10)$ & 82 (11) & $82(10)$ & 0.95 \\
\hline RR (SD) /min & $16(2)$ & $16(2)$ & $16(2)$ & 0.80 \\
\hline $\mathrm{BT}(\mathrm{SD}){ }^{\circ} \mathrm{C}$ & $37.3(1.0)$ & $37.4(1.1)$ & $37.2(0.9)$ & 0.19 \\
\hline WBC (SD) $\mathrm{k} / \mathrm{mm}^{3}$ & $13.2(3.9)$ & $12.9(4.2)$ & $13.4(3.7)$ & 0.31 \\
\hline Ultrasound (\%) & $51(18.3)$ & $22(19.1)$ & $29(17.7)$ & 0.87 \\
\hline $\mathrm{CT}$ scan $(\%)$ & $22(7.9)$ & $10(8.7)$ & $12(7.3)$ & 0.62 \\
\hline
\end{tabular}


Table 3 Time to operation and rate of perforated appendicitis

\begin{tabular}{|c|c|c|c|c|}
\hline & Total $(n=279)$ & Group $1(n=115)$ & Group $2(n=164)$ & p Value \\
\hline Mean time to operation (SD) min & $1012.7(650.3)$ & $831.6(582.5)$ & $1134.2(605.0)$ & 0.71 \\
\hline $\begin{array}{l}\text { Median time to operation } \\
\text { (interquartile range) min }\end{array}$ & $430(279-759)$ & $390(259-608)$ & $545(303-913)$ & 0.001 \\
\hline Perforated appendicitis (\%) & $60(21.5)$ & $25(21.7)$ & $35(21.3)$ & 0.88 \\
\hline
\end{tabular}

surgery is significantly shorter in the group who received analgesia $(390 v 545$ minutes, $\mathrm{p}=0.001)$. Preconsultation use of parenteral analgesics has not caused significant delay to surgery and is not associated with an increased rate of perforated appendicitis.

What is of interest and has been shown previously is that the time to surgery was shorter in the patients who received analgesia. A reasonable explanation is that these patients who needed early pain control had greater abdominal pain and would have decided for surgery earlier than those who had less abdominal pain. As appendicectomy is not an emergent operation, the decision for appendicectomy is usually an informed consent agreement between surgeons and patients.

There are several limitations to this study. Firstly, not all patients with suspected appendicitis were included in the study. Only those patients with surgically confirmed appendicitis were identified. The rate of false-positive surgery was not taken into consideration. Secondly, the retrospective design compromised the ability to reliably collect all data points resulting in the elimination of many cases. Thirdly, the representativeness of our database is limited as our population represents a selected group of patients only and may not applicable to other locales. In the future, a prospective randomised controlled study with larger study population would be needed to confirm our findings.

\section{CONCLUSION}

Pain control in ED patients with acute abdominal pain has remained controversial. Our study demonstrates that the preconsultation use of analgesics in ED patients with a final diagnosis of appendicitis is not associated with a longer delay to operative intervention and is not associated with an increased rate of perforated appendicitis.

\section{Contributors}

CC Chen and HP Ma collected the data. CF Chong organised and interpreted the data, and wrote the paper. TL Wong and H Chang supervised the study.

\section{Authors' affiliations}

C-F Chong, T-L Wang, C-C Chen, H-P Ma, H Chang, Emergency Department, Shin Kong Wu Ho-Su Memorial Hospital, Taipei, Taiwan Funding: none.

Conflicts of interest: none declared.

\section{REFERENCES}

1 Addiss DG, Shaffer N, Fowler BS, et al. The epidemiology of appendicitis and appendectomy in the United States. Am J Epidemiol 1990;132:910-25.

2 Trautlein J, Lambert R, Miller J. Malpractice in the emergency departmentreview of 200 cases. Ann Emerg Med 1984; 13:709-11.

3 Rusnak R, Borer J, Fastow J. Misdiagnosis of acute appendicitis: common features discovered in cases after litigation. Am J Emerg Med 1994; 12:397-402.

4 Cope Z, ed. Early diagnosis of the acute abdomen. 14th edn. New York: Oxford University Press, 1972:5-6.

5 Lovecchio F, Oster N. The use of analgesics in patients with acute abdominal pain. J Emerg Med 1997; 15:775-9.

6 Pace S, Burke T. Intravenous morphine for early pain relief in patients with acute abdominal pain. Acad Emerg Med 1996;3:1086-92.

7 Attard AR, Corlett MJ, Kidner NJ, et al. Safety of early pain relief for acute abdominal pain. BMJ 1992;305:554-6.

8 Graber MA, Ely JW. Informed consent and general surgeons' attitudes toward the use of pain medication in the acute abdomen. Am J Emerg Med 1999;17:113-16.

9 Greenfield RH, Henneman PL. Disorders of the small intestine. In: Rosen P, ed. Emergency medicine concepts and clinical practice. St Louis, MO: Mosby, 1998:2005-22.

10 Tait IS, lonescu MV, Cuschieri A. Do patients with acute abdominal pain wait unduly long for analgesia? J R Coll Surg Edinb 1999:44:181-4.

11 McHale PM, LoVecchio F. Narcotic analgesia in the acute abdomen-a review of prospective trials. Eur J Emerg Med 2001;8:131-6.

12 Mahadevan M, Graff L. Prospective randomized study of analgesic use for ED patients with right lower quadrant abdominal pain. Am J Emerg Med 2000;18:753-6. 